\title{
Both Hair Cortisol Levels and Perceived Stress Predict Increased Symptoms of Depression: An Exploratory Study in Young Adults
}

\author{
Markus Gerber $^{\mathrm{a}}$ Nadeem Kalak ${ }^{\mathrm{b}}$ Catherine Elliot ${ }^{\mathrm{a}}$ Edith Holsboer-Trachsler ${ }^{\mathrm{b}}$ \\ Uwe Pühse $^{\text {a }}$ Serge Brand ${ }^{b}$ \\ anstitute of Exercise and Health Sciences, University of Basel, and b Psychiatric Hospital of the University of Basel, \\ Center for Affective, Stress and Sleep Disorders, Basel, Switzerland
}

\section{Key Words}

Depressive symptoms · Hair cortisol · Hypocortisolism .

Perceived stress · Physical activity · Young adults

\begin{abstract}
Objective: Depressive symptoms are a common mental health problem among young adults, but the physiological mechanisms that mediate between stress and depressive symptoms remain unclear. Accordingly, this exploratory study (1) examined how hair cortisol concentrations were associated with self-perceived stress and depressive symptoms in a sample of young adults and (2) tested whether hair cortisol could explain variance in depressive symptoms beyond perceived stress before and after controlling for levels of vigorous physical activity (VPA). Methods: The sample consisted of 42 exercise and health science university students ( 20 males, 22 females; mean age $=21.2$ years). Cortisol concentrations were extracted from hair strands close to the scalp. Participants completed self-rating questionnaires about depressive symptoms and perceived stress. Results: Students with elevated hair cortisol levels tended to report lower depressive symptoms and lower perceived stress. Increased perceived stress was associated with higher depressive symptoms, and both hair cortisol and perceived stress predicted depressive symptoms after controlling for VPA.
\end{abstract}

Conclusions: The present data suggest that elevated hair cortisol levels do not necessarily constitute a health risk. Hair cortisol measurement can serve as a noninvasive and painless biomarker of chronic stress and mental disorders; however, additional research is needed.

Copyright $\odot 2013$ S. Karger AG, Basel

\section{Introduction}

Depressive symptoms are a common mental health problem among young adults [1-3]. Among university students, lifetime prevalence rates of major depressive disorder (MDD) vary between 10 and 25\% [4-6], and the proportion of students who perceive themselves as having nonclinical depressive symptoms is even higher $[7,8]$.

While depressive symptoms are associated with selfperceived stress and exposure to stressful events [9-11], there is a dearth of knowledge regarding the physiological mechanisms that mediate between chronic stress and depressive symptoms [12]. Chronic stress alters the activity of the hypothalamo-pituitary-adrenocortical (HPA) axis, which contributes to the development of

M.G. and N.K. contributed equally to the manuscript.

\section{KARGER}

E-Mail karger@karger.com

www.karger.com/nps
(C) 2013 S. Karger AG, Basel

0302-282X/13/0682-0100\$38.00/0
Markus Gerber

Institute of Exercise and Health Sciences, University of Basel Birsstrasse 320B

$\mathrm{CH}-4052$ Basel (Switzerland)

E-Mail markus.gerber@unibas.ch 
psychiatric disorders such as depression, anxiety and burnout. Several studies revealed that both hyper- and hypocortisolism can have harmful consequences to mental health [13-16].

Hair cortisol measurement has the potential to overcome many of the methodological limitations $[17,18]$ associated with saliva, blood and urine measurements $[19,20]$. For instance, only weak relationships were observed between salivary, plasma and urinary cortisol concentrations and perceived stress and self-reported health [21]. It is possible that these measures represent point assessments and only reveal acute circulating cortisol levels or mean cortisol secreted over a relatively short period of time. Moreover, salivary and plasma cortisol are (1) easily affected by temporary or transient disturbances in psychosocial stress on the day of measurement, (2) subject to diurnal variations and (3) affected by any food intake, smoking and physical activity that takes place shortly before sampling [17, 20, 22-24]. By contrast, the cortisol level in human hair has the potential to serve as a reliable endogenous biomarker for the retrospective assessment of long-term central HPA axis activity $[18,25,26]$. Stalder et al. [27] have shown that hair cortisol concentrations have a high intraindividual stability, with correlations of repeated assessments between 2 months and 1 year ranging between 0.68 and 0.79 . Increased hair cortisol concentrations were found in patients with Cushing's syndrome [28, 29], in women during the third trimester of pregnancy $[19,24]$ and in endurance athletes compared to inactive controls [30]. Likewise, higher hair cortisol concentrations were found in long-term unemployed versus employed individuals [22], in shift workers versus day workers [31], in alcoholics with acute withdrawal versus abstinent alcoholics or controls [32], in preterm infants with a greater number of days using a ventilator [33] and in traumatized individuals with posttraumatic stress disorder (PTSD) versus without PTSD [34]. Van Uum et al. [20] further observed that patients with severe chronic pain had higher hair cortisol levels than healthy (nonobese) adults, and elevated hair cortisol concentrations were associated with an increased risk for myocardial infarctions $[18,35]$ and overweight/obesity [28, 31].

Regarding the association between hair cortisol levels and mental health, both hyper- and hypocortisolism have been observed. Steudte et al. [26] found decreased hair cortisol levels in patients suffering from general anxiety disorder compared to matched healthy controls. Conflicting results exist with respect to depressive symptoms; while medicated patients with depression had higher hair

Hair Cortisol, Perceived Stress and Depressive Symptoms cortisol levels than healthy controls [23], depression was not associated with increased levels of hair cortisol in cardiac rehabilitation patients [25]. Finally, Karlen et al. [12] showed that 2 of 4 statistical outliers with very high hair cortisol levels had serious psychological problems in a sample of young adults.

In summary, previous studies have shown that hair cortisol levels may represent a biological correlate of psychiatric disorders, but the direction of the relationship remains unclear. Moreover, patients with MDD proved to have higher hair cortisol levels than healthy controls, and increased hair cortisol concentrations were found in young adults with severe mental health problems compared to peers with 'inconspicuous' mental health profiles.

Thus, the purpose of this study was twofold. The first purpose was to find out whether participants with low and elevated hair cortisol concentrations differ in relation to depressive symptoms and perceived stress. The second purpose was to examine whether hair cortisol concentrations could explain the variance in depressive symptoms beyond the variance that stems from self-perceived stress. Based on the aforementioned research findings, our hypotheses were that participants with elevated hair cortisol levels would score higher on depressive symptoms (hypothesis 1a) and perceived stress (hypothesis $1 \mathrm{~b}$ ). We further expected a moderate to strong positive association between perceived stress and depressive symptoms (hypothesis $2 \mathrm{a}$ ), that perceived stress would be a stronger predictor of depressive symptoms than hair cortisol (hypothesis $2 \mathrm{~b}$ ) and that hair cortisol would explain a small proportion of variance in depressive symptoms beyond the level contributed by perceived stress before and after controlling for participants' levels of vigorous physical activity (VPA; hypothesis 2c). Controlling for VPA was deemed important because Skoluda et al. [30] recently discovered that endurance athletes have increased hair cortisol levels compared to controls and that there is a dose response relationship between training volume and hair cortisol levels. These findings suggest that repeated exercise-induced HPA axis activation following acute bouts of exercise above approximately $70 \%$ of maximal oxygen uptake [36] coincides with increased hair cortisol levels, which raises important issues regarding the complexity of interpreting cortisol data in leisure-time exercisers. In other words, it is possible that part of the retrospective cortisol level measured in young adults' hair is likely due to repeated cortisol elevations associated with VPA and, to a lesser extent, exposure to psychological stress [37]. 
The present explorative study adds to the extant literature by examining the relationship between hair cortisol and depressive symptoms in young adults who were recruited in a nonclinical setting. Therefore, it sheds light on the complex relationship between physiological (HPA axis) and psychological (depressive symptoms, perceived stress) processes among young adults. The results are important for psychiatrists, general practitioners, occupational health services and primary care physicians in judging whether hair cortisol can identify the risk of developing depressive symptoms in young people and, ultimately, suppress clinical depression [38].

\section{Methods}

\section{Participants and Procedure}

The participants were 46 undergraduate exercise and health science students from the University of Basel, Switzerland [26 women, 20 men; mean age $=21.17$ years, $S D=1.87$ ] who volunteered to take part in the study. All participants provided written informed consent and demographic data, including gender, age, depressive symptoms and levels of perceived stress. Due to incomplete data (see below), the final sample consisted of 42 participants.

Participants received a food voucher (worth CHF 12) as an incentive. The local ethics committee of Basel (EKBB) approved the study protocol, and the study was conducted in accordance with the Declaration of Helsinki.

\section{Hair Cortisol Analysis}

Hair strands of approximately $3 \mathrm{~mm}$ in diameter were taken from a posterior vertex position close to the scalp. Trained study personnel collected hair samples using fine-tipped surgical scissors. Cortisol concentrations were tested for the first $3-\mathrm{cm}$ hair segment most proximal to the scalp, providing a retrospective index of cortisol secretion over the past 3 months. Due to an insufficient amount of hair ( $<3 \mathrm{~cm}$ or $<25 \mathrm{mg})$, data from 3 participants were excluded from hair cortisol analyses. The data of 1 participant were excluded because the presence of corticosteroid medication substantially increased the hair cortisol concentrations (117.7 pg/ $\mathrm{mg}$ ) compared to peers $($ mean $=25.17 \mathrm{pg} / \mathrm{mg}, \mathrm{SD}=12.93$, range $=$ $6.89-63.67$; skewness $=0.07$, kurtosis $=-0.20$ ). The wash procedure and steroid extraction were carried out as described in the laboratory protocol of Kirschbaum et al. [19].

\section{Assessment of Depressive Symptoms}

The German version of the Beck Depression Inventory (BDI) [39] was used to assess the severity of depressive symptoms. The BDI consists of 21 items including a range of affective, behavioral, cognitive and somatic symptoms that are indicative of unipolar depression (e.g. 'I am so unhappy/sad that I can't stand it'). Each item contains 4 responses that reflect increasing levels of depressive symptomatology. Possible scores range from 0 to 63 , with higher scores indicating more depressive symptoms. Adequate validity and reliability of the BDI have been shown previously [40]. The Cronbach's $\alpha$ in the present study was 0.81 for the total sample (mean = $4.69, \mathrm{SD}=6.33$, range $=0-34$; skewness $=0.07$, kurtosis $=-0.73$ ).

\section{Assessment of Perceived Stress}

General perceived stress during the past month was assessed with a German version of the widely used Perceived Stress Scale (PSS) [41]. The PSS consists of 10 short items measuring the degree to which respondents find their lives unpredictable, uncontrollable and overloading (e.g. 'How often have you felt that you could not control the important things in your life?'). Answers were given on a 4-point Likert scale from 1 (never) to 4 (very often). Items were summed to obtain an overall score. Adequate validity and reliability of this instrument have been established previously [42]. The Cronbach's a in the present study was 0.86 for the total sample ( mean $=22.83, \mathrm{SD}=5.95$, range $=14-40$; skewness $=0.73$, kurtosis $=0.27)$.

\section{Assessment of VPA}

VPA was assessed with an accelerometer (GT1M, Actigraph, Shalimar, Fla., USA) worn around the hip. The measurement period lasted for 7 consecutive days $[43]^{1}$, and the sampling epoch was set at $15 \mathrm{~s}$ [44]. Participants were instructed not to wear the accelerometer during the night. Time spent in VPA each day was determined based on the raw accelerometer counts and the ActiLife $^{\circledR}$ computer software, with cutoff values derived from Freedson et al. [45]. All activities with $>5,724$ counts/min were defined as vigorous, which corresponds to activities with an intensity level of $>6$ metabolic equivalents of task ( 1 metabolic equivalent of task corresponds to the metabolism at rest of an average person). Evidence of the reliability and validity of the Actigraph instruments has been published previously [46-48].

\section{Statistical Analysis}

A median split was conducted to separate participants into 2 groups, i.e. those with low hair cortisol levels (LHC; mean $=15.66$ $\mathrm{pg} / \mathrm{ml}, \mathrm{SD}=3.53$ ) and those with high hair cortisol levels (HHC; mean $=35.63 \mathrm{pg} / \mathrm{ml}, \mathrm{SD}=11.26) .^{2}$ Analyses of variance were used to compare LHC and HHC students with regard to depressive symptoms and perceived stress. The level of probability was set at $\mathrm{p}<0.05$. Due to the small sample size, results that tended to be significant $(p<0.10)$ were also demarcated, and we used $\eta^{2}$ as an orientation to interpret the strength of the relationships. Cohen [49] recommends that $\eta^{2}$ values from 0.010 to $<0.059$ should be interpreted as small associations, those from 0.059 to $<0.138$ as medium associations and those from 0.138 as large associations.

\footnotetext{
${ }^{1}$ This reference period was shorter than the 3-month time period for which the hair segment was collected. However, all participants indicated that their activity level during the assessment period was representative of their usual exercise behavior.

${ }^{2}$ Although criticized previously [87], relying on a statistical cutoff criterion to build groups seemed legitimate in the present study, as no knowledge exists about what 'low' and 'high' cortisol levels mean from a health or clinical point of view. A $\chi^{2}$ test showed that the LHC and HHC groups did not differ in terms of gender $\left[\chi^{2}(1,42)=0.89, p=0.346\right]$. Analyses of variance revealed that the LHC and HHC groups did not differ with regard to age $[\mathrm{F}(1,41)=$ $0.01, p=0.916]$. Analysis of variance further revealed no significant difference for VPA $[F(1,41)=2.37, p=0.132]$. Nevertheless, a slight tendency (5.7\% of variance explained) existed, showing that the LHC group exhibited lower VPA compared to the HHC group (mean $=55.74 \mathrm{~min} /$ week, $\mathrm{SD}=45.5$ vs. mean $=82.9 \mathrm{~min} /$ week, $\mathrm{SD}=66.1$ ). Therefore, both uncontrolled and controlled analyses were performed to rule out the possibility that participants' levels of VPA contributed to the differences in depressive symptoms.
} 
To find out whether and to what degree hair cortisol levels and self-perceived stress independently predict depressive symptoms, we conducted structural equation modelling (SEM) with Amos 20.0 (refer to Byrne [50] and Blunch [51] for a general introduction to the SEM approach). SEM has the ability to control for measurement error and considers that hair cortisol and perceived stress (as predictors of depressive symptoms) might be intercorrelated [50]. Correlations and regression weights were interpreted using Cohen's [49] criteria (i.e. small $<0.30$, medium $0.30-0.50$, large $>0.50$ ). SEM was based on maximum likelihood estimations and performed with and without controlling for VPA. The following indicators were used to evaluate the model fit: ratio between minimum discrepancy and degrees of freedom ( $\mathrm{cmin} / \mathrm{df})$, adjusted goodness-of-fit index (AGFI), comparative fit index (CFI), normed fit index (NFI) and root mean square error of approximation (RMSEA) [52]. ${ }^{3}$

\section{Results}

Depressive Symptoms and Perceived Stress Dependent upon Participants' Hair Cortisol Levels

The LHC group reported more depressive symptoms (mean BDI score $=6.23, \mathrm{SD}=7.89$ ) than the HHC group (mean BDI score $=3.00, \mathrm{SD}=3.45$ ). The difference tended to be statistically significant $[\mathrm{F}(1,41)=2.85, \mathrm{p}=0.099]$, with $6.6 \%$ of variance explained. After controlling for VPA, the previously observed tendency disappeared $[\mathrm{F}(1$, $41)=1.25, \mathrm{p}=0.271]$, but the effect size still pointed towards a small effect $\left(\eta^{2}=0.032\right)$. The LHC group also reported higher perceived stress (mean PSS score $=24.23$, $\mathrm{SD}=6.87$ ) than the HHC group (mean PSS score $=21.30$, $\mathrm{SD}=4.41)$. Even though the difference was not statistically significant $[\mathrm{F}(1,41)=2.64, \mathrm{p}=0.112]$, the level of explained variance reached $6.2 \%$. The effect size only decreased slightly after controlling for $\operatorname{VPA}[\mathrm{F}(1,41)=2.07$, $\left.p=0.159, \eta^{2}=0.050\right]$. Finally, it is noteworthy that those participants who reported mild depression $(n=2$, BDI scores between 11 and 17) or clinically relevant depression $(\mathrm{n}=2$, BDI scores $\geq 18)$ were all classified into the LHC group.

A median split based on the BDI further showed that participants with lower depression scores had slightly higher hair cortisol concentrations (mean $=28.35 \mathrm{pg} /$ $\mathrm{mg}, \mathrm{SD}=15.48)$ than participants with higher depres-

\footnotetext{
${ }^{3}$ Several 'rules of thumb' exist regarding the minimum sample size required for the estimation of structural equation models to achieve a satisfactory model fit $[88,89]$. Scholars generally agree that the risk of rejecting a true model is lower if the sample size is large [90]. However, it has been argued that the effect of sample size on some fit statistics such as CFI is nonlinear and that therefore smaller samples suffice if the variables are reliable and the model is not overly complex [91].
}

Hair Cortisol, Perceived Stress and Depressive Symptoms sion scores [mean $=21.98 \mathrm{pg} / \mathrm{mg}, \mathrm{SD}=98.04 ; \mathrm{F}(1,41)=$ $\left.2.65, \mathrm{p}=0.112, \eta^{2}=0.062\right]$. Nevertheless, although the effect size was of moderate magnitude, the group difference was not statistically significant due to the limited sample size.

Higher hair cortisol concentrations were also found in participants with low versus high PSS scores (based on a median split on the PSS). Nevertheless, the group differences were not statistically significant $[\mathrm{F}(1,41)=0.81, \mathrm{p}=$ $\left.0.37, \eta^{2}=0.020\right]$. The mean hair cortisol concentration in the lower PSS group was $27.15 \mathrm{pg} / \mathrm{mg}(\mathrm{SD}=13.46)$, compared to $23.53 \mathrm{pg} / \mathrm{mg}(\mathrm{SD}=12.57)$ in the higher PSS group.

\section{Hair Cortisol Level and Perceived Stress as Predictors} of Depressive Symptoms

Figure 1 shows the results of the SEM, with depressive symptoms as the outcome variable, which is predicted by participants' self-perceived stress and their hair cortisol levels. With respect to the criteria (in parentheses below) proposed by McDonald and Ho [52], the model represented a satisfactory fit with regard to most indices: $\mathrm{cmin} /$ $\mathrm{df}=2.66, \mathrm{AGFI}=0.89(\geq 0.95), \mathrm{CFI}=0.98(>0.90), \mathrm{NFI}=$ $0.96(>0.90)$ and RMSEA $=0.10(\leq 0.05){ }^{4}$

Figure 1a shows that self-perceived stress and objectively assessed hair cortisol tended to be weakly correlated $(\mathrm{r}=-0.18, \mathrm{p}=0.052)$. The negative sign corroborates the finding that participants with increased hair cortisol levels reported lower perceived stress. In contrast, self-perceived stress was a strong positive predictor of depressive symptoms $(\beta=0.79, \mathrm{p}=0.000)$, indicating that high perceived stress was associated with more depressive symptoms. Although self-perceived stress explained a substantial amount of variance in depressive symptoms, a weak but significant association emerged for hair cortisol levels. Again, the negative direction of the relationship suggests that increased hair cortisol concentrations were negatively associated with depressive symptoms $(\beta=-0.24, p=0.009)$. The total amount of variance explained through perceived stress and hair

\footnotetext{
${ }^{4}$ Perceived stress and depressive symptoms were operationalized as latent variables (represented by ellipses). Both latent factors were predicted by $2 \mathrm{ob}-$ servable variables (randomly built parcels with half of the items; represented by rectangles) [50]. A parcel can be defined as an aggregate-level indicator comprised of the sum (or average) of 2 or more items, responses or behaviors [92]. The main reason for building parcels is to keep structural equation models simple by reducing their degree of freedom $[50,93,94]$. Comrey and Lee [95] recommended to interpret standardized factor loadings (pointing from latent factors to observed variables) of $>0.71$ as excellent, $>0.63$ as very good, $>0.55$ as good, $>0.45$ as fair and $>0.32$ as poor. Thus, all factor loadings were good in the present analysis.
} 

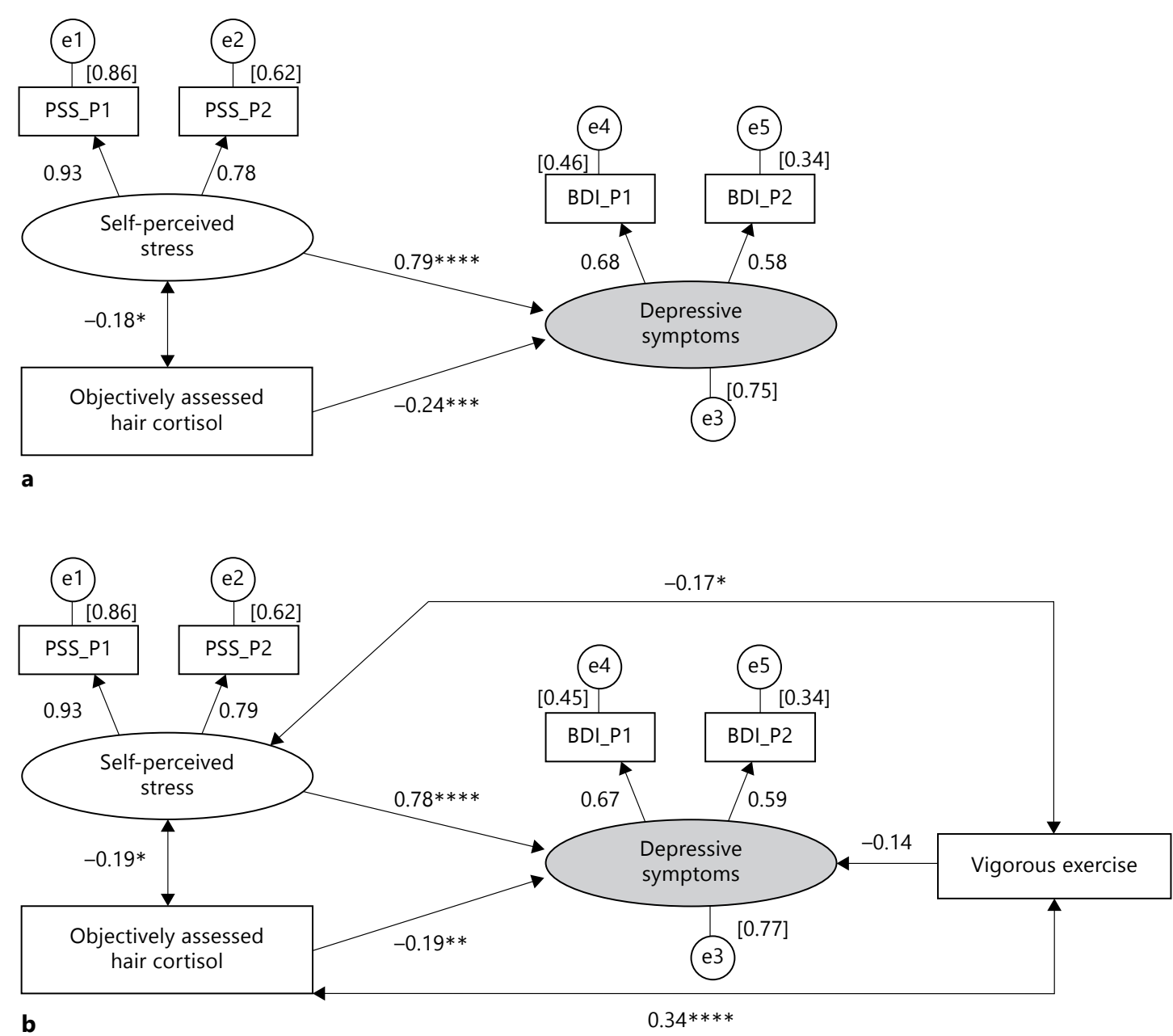

Fig. 1. Hair cortisol and perceived stress as predictors of self-reported depressive symptoms. Text in ellipses describes latent factors, while that in rectangles refers to observed variables. Coefficients in square brackets show squared multiple correlations, while coefficients without brackets represent correlations or standardized regression weights. ${ }^{*} \mathrm{p}<0.10$; $^{* *} \mathrm{p}<0.05$; $^{* * *} \mathrm{p}<0.01$; $^{* * * *} \mathrm{p}<$ 0.001. e $=$ Measurement errors; $\mathrm{P} 1=$ parcel 1; P2 $=$ parcel 2.

unchanged $(\beta=-0.78, p=0.000)$. The strength of the negative relationship between hair cortisol and depressive symptoms decreased slightly $(\beta=-0.19, \mathrm{p}=0.048)$ but was still statistically significant.

\section{Discussion}

Three findings of the present study in young adults deserve special emphasis. Firstly, although not statistically significant, participants with elevated levels of hair cortisol tended to report lower depressive symptoms and lower perceived stress. Secondly, increased self-perceived 
stress was associated with higher depressive symptoms. Thirdly, both objectively assessed hair cortisol levels and self-reported stress significantly explained variance in depressive symptoms.

Five hypotheses were formulated and each of these will now be discussed separately. We expected that elevated hair cortisol levels would be associated with increased depressive symptoms (hypothesis 1a), although our data did not support this assumption. Rather, increased hair cortisol levels were associated with lower depressive symptoms. This finding is surprising for at least 4 reasons. Firstly, Jonsdottir et al. [53] recently published a review on the relationship between salivary cortisol and mental health including MDD and symptoms of depression in otherwise healthy participants. Using a broad range of cortisol measures, the authors concluded that individuals diagnosed with MDD typically have higher salivary cortisol levels. The results are less consistent for nonclinical depressive symptoms but still point in a similar direction. Secondly, studies have shown that higher plasma cortisol concentrations are associated with increased depressive symptoms in both healthy and clinical populations [54-58] and that plasma cortisol levels can decrease in patients with MDD after pharmacotherapy $[59,60]$. Thirdly, Dettenborn et al. [23] found that patients with MDD had higher hair cortisol concentrations in both the first and second $3-\mathrm{cm}$ hair segments compared to age- and gender-matched healthy controls. Fourthly, Karlen et al. [12] pointed out in a study with young adults that out of 4 outliers with extremely high hair cortisol concentrations, 2 reported serious psychological problems including severe generalized anxiety disorder with panic attacks. Thus, although at first glance the negative relationship between hair cortisol level and depressive symptoms seems puzzling in light of the above findings, evidence exists that the association between hair cortisol and depression might not be straightforward. Dowlati et al. [25] did not find differences in hair cortisol between patients with coronary heart disease independent of the presence of MDD. Similarly, Stalder et al. [32] were unable to demonstrate a significant relationship between hair cortisol and the BDI score in a combined sample of abstinent and nonabstinent alcoholics and healthy controls. Notwithstanding these findings, very little research exists among young healthy adults, and the few existing hair cortisol studies were not explicitly focused on mental health outcomes $[12,27,28,61]$. Among the studies that correlated hair cortisol levels with mental health indicators, only 1 investigation suggested that mental disorders may be associated with decreased hair cortisol levels [26]. This study indicated that participants suffering from gen-

Hair Cortisol, Perceived Stress and Depressive Symptoms eralized anxiety disorder had significantly lower (50-60\%) hair cortisol concentrations compared to age- and gendermatched healthy controls. In addition, Skoluda et al. [30] revealed that regular exercisers had higher hair cortisol concentrations than inactive controls. This corroborates the notion that increased hair cortisol concentrations are not necessarily pathological, since a wealth of studies exist showing that regular exercise prevents the development of depressive symptoms $[62,63]$ and that exercise leads to positive outcomes in the treatment of $\operatorname{MDD}[64,65]$.

In summary, finding increased hair cortisol concentrations associated with decreased depressive symptoms in this exploratory study was unexpected. Although some associations only tended to be significant, this result suggests that increased hair cortisol levels are not necessarily 'bad' for young adults' health. This is in line with Hatzinger et al. [66] who showed that among young girls, high cortisol secretion during a laboratory stressor task was associated with positive emotions. Nevertheless, we explicitly emphasize that the findings of our study should be interpreted cautiously and in view of this study's limitations. Namely, the sample represents a very specific segment of the larger population comprising only undergraduate exercise and health science students without any known mental disorders. Moreover, all participants accomplished recommended levels of physical activity and had to pass a practical exam (sport and fitness test) to be accepted as a student in the exercise and health science institute. Our sample therefore represented a relatively homogeneous and healthy cohort. Nevertheless, the mean, SD and range of hair cortisol concentrations in our sample were comparable to those in previous studies with young adults and 'healthy' controls [12, 22, 23, 30]. Moreover, the differences in depressive symptoms found between the students with low and elevated hair cortisol levels only tended to be significant. Although hair cortisol provides a retrospective window to the past, this study was cross-sectional and prohibits causal inferences. Finally, data obtained from larger samples are needed to reduce the possible impact of outliers on the relationship between hair cortisol and mental health indicators. Larger samples would also allow a comparison of more extreme groups, which might result in bigger group differences.

With the second hypothesis (hypothesis $1 \mathrm{~b}$ ), we anticipated that increased hair cortisol levels would be associated with increased self-perceived stress, although our data failed to support this assumption. Significant correlations have been found between hair cortisol and self-reported stress among chronic pain patients [20] and 
pregnant women [67]. However, the correlations found in these studies pointed towards a moderate positive relationship, while we found a negative association. So far, only 1 study exists with a comparable sample of healthy young adults [12]. Similar to our study, a weak negative correlation existed between hair cortisol and the score on the PSS $(\mathrm{r}=-0.06, \mathrm{p}=0.025)$. In contrast, Karlen et al. [12] found a stronger (positive) relationship for exposure to serious life events in a regression model $(\beta=0.21, \mathrm{p}=$ 0.041 ). The stronger association between hair cortisol and life events supports previous research showing that serious stressful events are associated with increased hair cortisol levels [22,33]. Several explanations are possible as to why hair cortisol concentrations show closer association with life events than perceived stress. For instance, researchers have attributed the weak relationship between perceived stress and hair cortisol to a 'lack of psychoendocrine covariance' known from studies where acute saliva cortisol reactions were dissociated from self-reported tensions or heart rate [68]. Moreover, different time frames assessed via hair cortisol and stress questionnaires may account for the nonsignificant relationships in the above studies. In our opinion, 2 further methodological issues should be considered. Firstly, life events are mostly studied from a between-group perspective, in which individuals who were exposed to a stressor (e.g. long-term unemployment) are compared with healthy controls. Comparing such 'extreme' groups might result in a relatively high variation in terms of stress. In contrast, the relationship between hair cortisol and perceived stress is mostly studied from a within-group perspective, in which hair cortisol and perceived stress are correlated in relatively homogeneous populations such as patients with coronary artery disease [25], women participating in a preterm birth study [69] or endurance athletes [30]. Within this approach, low correlation coefficients must be interpreted with caution as the lacking associations can be due to low variations in terms of stress and hair cortisol. Secondly, it is conceivable that life event lists have per se a higher discriminative power as only few individuals are faced with major life events, whereas most individuals encounter minor daily stressors [70, 71].

The remaining set of hypotheses was fully supported via SEM. A strong positive association existed between perceived stress and depressive symptoms (hypothesis 2a). Perceived stress was a stronger predictor of depressive symptoms than hair cortisol (hypothesis 2b). Finally, hair cortisol explained a small proportion of variance in depressive symptoms beyond that which can be ascribed to perceived stress (hypothesis $2 \mathrm{c}$ ). However, significant relationships emerged for all hypothesized paths in our SEM model. The fact that perceived stress was more strongly associated with depressive symptoms can be attributed to the fact that the consequences of stress largely depend on individual appraisals [72, 73]. Alternatively, a shared method variance might have contributed to the strong association between perceived stress and depressive symptoms.

In line with previous studies that have shown low correlations between biological markers of stress and mental health [74-76], hair cortisol turned out to be a significant predictor of depressive symptoms in the present study and explained additional variance beyond perceived stress. As mentioned above, the negative direction of the relationship was surprising and deserves further discussion. In our sample of young adults, the findings corresponded with research showing that some stress-related states and functional somatic diseases are associated with hypocortisolism [77]. Hypocortisolism is generally defined as a relative or absolute state of paradoxically low cortisol, which can manifest as inadequate basal cortisol production, flattened daytime cortisol production or inappropriate elevation of cortisol under stressful conditions [78]. In prior research, correlations were found between hypocortisolism and metabolic syndrome, fibromyalgia, chronic fatigue syndrome, occupational burnout, chronic pain syndromes, PTSD, cardiometabolic disease, mood disorders, autoimmune diseases, allergies and malignancies [78-82]. Although previous salivary cortisol studies have shown that depressive symptoms are typically associated with hypercortisolism [53], there is a strong comorbidity between depression and symptoms of burnout, pain, fatigue and exhaustion, which proved to be associated with hypercortisolism [83, 84]. Finally, Murck [85] pointed out that atypical depression with reversed vegetative signs (i.e. hyperphagia or hypersomnia) is typically associated with decreased cortisol-releasing hormone activity. While major depression is usually characterized by symptoms of depressed mood and loss of interest and pleasure (corresponding with melancholic features), it is possible that atypical forms of depression are particularly likely among exercise and health science students. This may explain the negative relationship between hair cortisol and depressive symptoms in this particular population.

In summary, our findings support the notion that perceived stress more strongly predicts depressive symptoms than objectively assessed hair cortisol levels. While hair cortisol predicted variance beyond perceived stress, this finding must be interpreted with caution because of the unique characteristics of this sample. Nevertheless, the 
present study suggests that the relationships between hair cortisol and mental health might be more complex than expected and that elevated hair cortisol might not always equate to a health risk in certain populations. Currently, research on the relationship between hair cortisol and mental health is in its infancy. Future studies should focus on how hair cortisol can be used as a comprehensive means to identify individuals at risk for somatic and mental diseases. Future studies should also work towards proofs of causality. In line with Kudielka and Wüst [86], who underscored in their recent review that both acute and chronic stress generally precede the onset of a broad variety of diseases such as depressive disorders, depressive symptoms were treated as a consequence in the present study. Nevertheless, it is conceivable that premorbid and preliminary propensity to depressive symptoms (cognitions, emotions, behavior) may unfavorably influence both HPA axis activity and individuals' perception of stress.

To the best of our knowledge, this is the first study indicating that low hair cortisol might be associated with increased depressive symptoms among young adults. More research is needed to corroborate this finding using larger samples and other indicators of stress and psychological functioning. We also recommend considering VPA more thoroughly in future research. Endurance athletes have higher hair cortisol levels than physically inactive controls [30]. A similar result was found in the present study, and the strength of associations between hair cortisol and depressive symptoms decreased after controlling for VPA.

\section{Conclusion}

The data of the present study point towards the potential role of low cortisol levels in the development of depressive symptoms in young adults. Edwards et al. [78] maintained that "traditional" medical practice does not sufficiently recognize relatively low cortisol levels as a potential cause of disease' (p. 27). If the findings of the present study can be confirmed in larger samples, hair cortisol might be useful for psychiatrists and general practitioners as a noninvasive and painless biomarker to identify young people at risk for developing stress-related diseases or functional somatic disorders associated with hypocortisolism.

\section{Acknowledgements}

The entire study was conducted without external funding. The authors alone are responsible for the content and writing the paper. We thank Lucia Bühlmayer, Andrea Glantschnig, Ramona Graf, Stefanie Häfeli, Christian Herrmann, Annemie Kostezer and Elvis Maccarone for their contribution to recruitment of participants, data collection and data processing. Finally, we thank all participants for their valuable time and effort. We are particularly grateful to the hair cortisol lab of Prof. Clemens Kirschbaum (Technische Universität Dresden, Germany) for hair cortisol analysis.

\section{Disclosure Statement}

There are no conflicts of interest to declare.

\section{References}

$>1$ Buysse DJ, Angst J, Gamma A, Ajdacic V, Eich D, Rossler W: Prevalence, course, and comorbidity of insomnia and depression in young adults. Sleep 2008;31:473-480.

$\checkmark 2$ Smith DJ, Harrison N, Muir W, Blackwood $\mathrm{DH}$ : The high prevalence of bipolar spectrum disorders in young adults with recurrent depression: toward an innovative diagnostic framework. J Affect Disord 2005;84:167-178.

-3 Aalto-Setala T, Marttunen M, Tuulio-Henriksson A, Poikolainen K, Lonnqvist J: Onemonth prevalence of depression and other DSM-IV disorders among young adults. Psychol Med 2001;31:791-801.

4 Roh MS, Jeon HJ, Kim H, Han SK, Hahm BJ: The prevalence and impact of depression among medical students: a nationwide crosssectional study in South Korea. Acad Med 2010;85:1384-1390.

$>5$ Tomoda A, Mori K, Kimura M, Takahashi T, Kitamura T: One-year prevalence and inci- dence of depression among first-year university students in Japan: a preliminary study. Psychiatry Clin Neurosci 2000;54:583-588.

6 Vazquez FL, Blanco V: Prevalence of DSM-IV major depression among Spanish university students. J Am Coll Health 2008;57:165-171.

7 Chang E, Eddins-Folensbee F, Coverdale J: Survey of the prevalence of burnout, stress, depression, and the use of supports by medical students at one school. Acad Psychiatry 2012;36:177-182.

8 Mikolajczyk RT, Maxwell AE, El Ansari W, Naydenova V, Stock C, Ilieva S, Dudziak U, Nagyova I: Prevalence of depressive symptoms in university students from Germany, Denmark, Poland and Bulgaria. Soc Psychiatry Psychiatr Epidemiol 2008;43:105-112.

$\checkmark 9$ Brand S, Gerber M, Pühse U, HolsboerTrachsler E: Depression, hypomania and dysfunctional cognitions as mediators between stress and insomnia: the best advice is not al- ways found on the pillow! Int J Stress Manag 2010;17:114-134.

10 Sawatzky RG, Ratner PA, Richardson CG, Washburn C, Sudmant W, Mirwaldt P: Stress and depression in students: the mediating role of stress management self-efficacy. Nurs Res 2012;61:13-21.

11 Dahlin M, Joneborg N, Runeson B: Stress and depression among medical students: a crosssectional study. Med Educ 2005;39:594-604.

-12 Karlen J, Ludvidsson J, Frostell A, Theodorsson E, Faresjo T: Cortisol in hair measured in young adults - a biomarker of major life stressors? BMC Clin Pathol 2011;11:12.

13 McEwen BS: Protective and damaging effects of stress mediators. N Engl J Med 1998;338: 171-179.

14 Miller G, Chen E, Zhou E: If it goes up, must it come down? Chronic stress and the hypothalamic-pituitary-adrenocortical axis in humans. Psychol Bull 2007;133:25-45.
Hair Cortisol, Perceived Stress and Depressive Symptoms
Neuropsychobiology 2013;68:100-109 DOI: $10.1159 / 000351735$ 
15 Chrousos GP: Stress and disorders of the stress system. Nat Rev Endocrinol 2009;5: 374-381.

-16 Tsigos C, Chrousos GP: Hypothalamic-pituitary-adrenal axis, neuroendocrine factors and stress. J Psychosom Res 2002;53:865-871.

17 Stalder T, Kirschbaum C: Analysis of cortisol in hair - state of the art and future directions. Brain Behav Immun 2012;26:1019-1029.

18 Gow R, Thomson S, Rieder M, van Uum S, Koren G: An assessment of cortisol analysis in hair and its clinical applications. Forensic Sci Int 2010;196:32-37.

19 Kirschbaum C, Tietze A, Skoluda N, Dettenborn L: Hair as a retrospective calendar of cortisol production - increased cortisol incorporation into hair in the third trimester of pregnancy. Psychoneuroendocrinology 2009; 34:32-37.

20 van Uum S, Sauvé B, Fraser LA, Morley-Forster P, Paul TL, Koren G: Elevated content of cortisol in hair of patients with severe chronic pain: a novel biomarker for stress. Stress 2008 ; 11:483-488.

21 Kristenson M, Garvin P, Lundberg U (eds): The Role of Saliva Cortisol Measurement in Health and Disease. Oak Park, Bentham Science Publishers, 2012.

22 Dettenborn L, Tietze A, Bruckner F, Krischbaum C: Higher cortisol content in hair among long-term unemployed individuals compared to controls. Psychoneuroendocrinology 2010;35:1404-1409.

-23 Dettenborn L, Muhtz C, Skoluda N, Stalder T, Steudte S, Hinkelmann K, Kirschbaum C, Otte C: Introducing a novel method to assess cumulative steroid concentrations: increased hair cortisol concentrations over 6 months in medicated patients with depression. Stress 2012;15:348-353.

24 D'Anna-Hernandez KL, Ross RG, Natvig CL, Laudenslager ML: Hair cortisol levels as a retrospective marker of hypothalamic-pituitary axis activity throughout pregnancy: comparison to salivary cortisol. Physiol Behav 2011; 104:348-353.

25 Dowlati Y, Herrmann N, Swardfager W, Thomson S, Oh PI, van Uum S, Koren G, Lactôt K: Relationship between hair cortisol concentrations and depressive symptoms in patients with coronary artery disease. Neuropsychiatr Dis Treat 2010;6:393-400.

26 Steudte S, Stalder T, Dettenborn L, Klumbies E, Foley P, Beesdo-Baum K, Kirschbaum C: Decreased hair cortisol concentrations in generalised anxiety disorder. Psychiatry Res 2011;186:310-314.

-27 Stalder T, Steudte S, Miller R, Skoluda N, Dettenborn L, Krischbaum C: Intraindividual stability of hair cortisol concentrations. Psychoneuroendocrinology 2012;37:602-610.

28 Manenschijn L, Koper JW, Lamberts SWJ, van Rossum EFC: Evaluation of a method to measure long term cortisol levels. Steroids 2011;76:1032-1036.

29 Thomson S, Koren G, Fraser LA, Rieder M, Friedman TC, Van Uum SH: Hair analysis provides a historical record of cortisol levels in Cushing's syndrome. Exp Clin Endocrionol Diabetes 2010;118:133-138.

30 Skoluda N, Dettenborn L, Stalder T, Kirschbaum C: Elevated hair cortisol concentrations in endurance athletes. Psychoneuroendocrinology 2012;37:611-617.

-31 Manenschijn L, van Kruysbergen RGPM, de Jong FH, Koper JW, van Rossum EFC: Shift work at young age is associated with elevated long-term cortisol levels and body mass index. J Clin Endocrinol Metab 2011;96:E1862E1865.

32 Stalder T, Kirschbaum C, Heinze K, Steudte S, Foley P, Tietze A, Dettenborn L: Use of hair cortisol analysis to detect hypercortisolism during active drinking phases in alcohol-dependent individuals. Biol Psychol 2010;85: 357-360.

33 Yamada J, Stevens B, de Silva N, Gibbins S, Beyene J, Taddio A, Newman C, Koren G: Hair cortisol as a potential biologic marker of chronic stress in hospitalized neonates. Neonatology 2007;92:42-49.

34 Steudte S, Kolass I-T, Stalder T, Pfeiffer A, Kirschbaum C, Elbert T: Increased cortisol concentrations in hair of severely traumatized Ugandan individuals with PTSD. Psychoneuroendocrinology 2011;36:1193-1200.

35 Pereg D, Gow R, Mosseri M, Lishner M, Rieder M, van Uum S, Koren G: Hair cortisol and the risk for acute myocardial infarction in adult men. Stress 2011;14:73-81.

36 Borer K: Exercise Endocrinology. Champaign, Human Kinetics, 2003.

37 Gerber M, Brand S, Lindwall M, Elliot C, Kalak N, Herrmann C, Pühse U, Jonsdottir IH: Concerns regarding hair cortisol as a biomarker of chronic stress in exercise and sport science. J Sports Sci Med 2012;11:571-581.

38 American Psychiatric Association: DSM-IV. Diagnostic and Statistical Manual of Mental Disorders, ed 4. Washington, American Psychiatric Association, 2000.

39 Beck AT, Ward CH, Mendelson M, Mock J, Erbaugh J: An inventory for measuring depression. Arch Gen Psychiatry 1961;4:561571.

40 Hautzinger M, Bailer M, Worall H, Keller F: Beck Depressions-Inventar. Bern, Huber, 1995.

41 Cohen S, Kamarck T, Mermelstein R: A global measure of perceived stress. J Health Soc Behav 1983;24:385-396.

42 Cohen S, Williamson GM: Perceived stress in a probability sample of the United States; in Spacapan S, Oskamp S (eds): The Social Psychology of Health. Newbury Park, Sage, 1988, pp 31-67.

43 Trost SG, Pate RR, Freedson PS, Sallis JF, Taylor WC: Using objective physical activity measures with youth: how many days of monitoring are needed? Med Sci Sports Exerc 2000;32:426-431.

44 Rowlands AV: Accelerometer assessment of physical activity in children: an update. Pediatr Exerc Sci 2007;19:252-266.
45 Freedson PS, Melanson E, Sirard J: Calibration of the Computer Science and Applications, Inc. accelerometer. Med Sci Sports Exerc 1998;30:777-781.

46 Leenders NY, Nelson TE, Sherman WM: Ability of different physical activity monitors to detect movement during treadmill walking. Int J Sports Med 2003;24:43-50.

47 Silva P, Mota J, Esliger D, Welk G: Technical reliability assessment of the Actigraph GT1M accelerometer. Meas Phys Educ Exerc Sci 2010;14:79-91.

48 Rothney MP, Apker GA, Song Y, Chen KY: Comparing the performance of three generations of ActiGraph accelerometers. J Appl Physiol 2008;105:1091-1097.

49 Cohen J: Statistical Power Analysis for the Behavioral Sciences. Mahwah, Erlbaum, 1988.

50 Byrne BM: Structural Equation Modeling with AMOS. Basic Concepts, Applications, and Programming. New York, Taylor and Francis, 2010.

51 Blunch N: Introduction to Structural Equation Modeling using IBM SPSS Statistics and AMOS. London, Sage, 2013.

52 McDonald RP, Ho RM: Principles and practice in reporting structural equation analyses. Psychol Methods 2002;7:65-69.

53 Jonsdottir IH, Halford C, Eek F: Mental health and salivary cortisol; in Kristenson M, Garvin P, Lundberg U (eds): The Role of Saliva Cortisol Measurement in Health and Disease. Oak Park, Bentham Science Publishers, 2012, pp 129-166.

54 Majumdar SK, Shaw GK, Bridges PK: Relationship between plasma cortisol concentrations and depression in chronic alcoholic patients. Drug Alcohol Depend 1984;14: 45-49.

55 Cohen MR, Pickar D, Extein I, Gold MS Sweeney DR: Plasma cortisol and beta-endorphin immunoreactivity in nonmajor and major depression. Am J Psychiatry 1984;141: 628-632.

56 Chodzko-Zajko WJ, O’Connor PJ: Plasma cortisol, the dexamethasone suppression test and depression in normal adult males. J Psychosom Res 1986;30:313-320.

57 Deuschle M, Weber B, Colla M, Depner M, Heuser I: Effects of major depression, aging and gender upon calculated diurnal free plasma cortisol concentrations: a re-evaluation study. Stress 1998;2:281-287.

58 Morris MC, Compas BE, Garber J: Relations among posttraumatic stress disorder, comorbid major depression, and HPA function: a systematic review and meta-analysis. Clin Psychol Rev 2012;32:301-315

-59 Piwowarska J, Chimiak A, Matsumoto $\mathrm{H}$, Dziklinska A, Radziwon-Zaleska M, Szelenberger W, Pachecka J: Serum cortisol concentration in patients with major depression after treatment with fluoxetine. Psychiatry Res 2012;198:407-411.

60 Holsboer F, Barden N: Antidepressants and hypothalamic-pituitary-andrenocortical regulation. Endocr Rev 1996;17:187-205. 
-61 Xie Q, Gao W, Li J, Qiao T, Jin J, Deng H, Lu $\mathrm{Z}$ : Correlation of cortisol in $1-\mathrm{cm}$ hair segment with salivary cortisol in human: hair cortisol as an endogenous biomarker. Clin Chem Lab Med 2011;49:1-6.

62 Daley A: Exercise and depression: a review of reviews. J Clin Psychol Med Settings 2008;15: 140-147.

63 De Moor MHM, Beem AL, Stubbe JH, Boomsma DI, De Geus EJC: Regular exercise, anxiety, depression and personality: a population-based study. Prev Med 2006;42:273279.

64 Mota-Pereira J, Silverio J, Carvalho S, Ribeiro JC, Fonte D, Ramos J: Moderate exercise improves depression parameters in treatmentresistant patients with major depressive disorder. J Psychiatr Res 2011;45:1005-1011.

-65 Blumenthal JA, Michael A, Babjak A: Exercise and pharmacotherapy in the treatment of major depressive disorder. Psychosom Med 2007;69:587-596.

-66 Hatzinger M, Brand S, Perren S, Von Wyl A, Von Klitzing K, Holsboer-Trachsler E: Hypothalamic-pituitary-adrenocortical (HPA) activity in kindergarten children: importance of gender and associations with behavioral/ emotional difficulties. J Psychiatr Res 2007; $41: 861-870$

67 Kalra S, Einarson A, Karaskov T, Van Uum S, Koren G: The relationship between stress and hair cortisol in healthy pregnant women. Clin Invest Med 2007;30:E103-E107.

- 68 Schlotz W, Kumsta R, Layes I, Entringer S, Jones A, Wust S: Covariance between psychological and endocrine responses to pharmacological challenge and psychosocial stress: a question of timing. Psychosom Med 2008;70 787-796.

69 Kramer MS, Lydon J, Séguin L, Goulet L, Kahn SR, McNamara H, Genest J, Dassa C, Chen MF, Sharma S, Meaney JJ, Thomson S, van Uum S, Koren G, Dahhou M, Lamoureux J, Platt RW: Stress pathways to spontaneous preterm birth: the role of stressors, psychological distress, and stress hormones. Am I Epidemiol 2009;169:1319-1326.

70 Giacobbi PR, Hausenblas HA, Frye N: A naturalistic assessment of the relationship between personality, daily life events, leisuretime exercise, and mood. Psychol Sport Exerc 2005;6:67-81.
71 Twisk JW, Snel J, Kemper HC, van Mechelen W: Changes in daily hassles and life events and the relationship with coronary heart disease risk factors: a 2-year longitudinal study in 27-29-year-old males and females. J Psychosom Res 1999;46:229-240.

72 Lazarus RS, Folkman S: Stress, Appraisal, and Coping. New York, Springer, 1984.

73 Hobfoll SE: Stress, Culture, and Community. The Psychology and Philosophy of Stress. New York, Plenum Press, 1998.

74 Eller NH, Kristiansen J, Hansen AM: Longterm effects of psychosocial factors of home and work on biomarkers of stress. Int J Psychophysiol 2011;79:195-202.

75 Persson R, Orbaek P, Kecklund G, Akerstedt T: Impact of an 84-hour workweek on biomarkers for stress, metabolic processes and diurnal rhythm. Scand J Work Environ Health 2006;32:349-358.

76 Chida Y, Steptoe A: Cortisol awakening response and psychosocial factors: a systematic review and meta-analysis. Biol Psychol 2009; 80:265-278

77 Tak LM, Cleare AJ, Ormel J, Manoharan A, Kok IC, Wessely S, Rosmalen JG: Meta-analysis and meta-regression of hypothalamic-pituitary-adrenal axis activity in functional somatic disorders. Biol Psychol 2011;87:183194.

78 Edwards LD, Heyman AH, Swidan S: Hypocortisolism: an evidence-based review. Int Med 2011;10:26-33.

79 Fries E, Hesse J, Hellhammer J, Hellhammer DH: A new view on hypocortisolism. Psychoneuroendocrinology 2005;30:1010-1016.

80 Heim C, Ehlert U, Hellhammer DH: The potential role of hypocortisolism in the pathophysiology of stress-related bodily disorders. Psychoneuroendocrinology 2000;25:1-35.

81 Raison CL, Miller AH: When not enough is too much: the role of insufficient glucocorticoid signaling in the pathophysiology of stress-related disorders. Am J Psychiatry 2003;160:1554-1565.

82 Yehuda R: Sensitization of the hypothalamicpituitary-adrenal axis in posttraumatic stress disorder. Ann NY Acad Sci 1997;821:57-75.
83 Ruehlman LS, Karoly P, Pugliese J: Psychosocial correlates of chronic pain and depression in young adults: further evidence of the utility of the Profile of Chronic Pain: Screen (PCP: S) and the Profile of Chronic Pain: Extended Assessment (PCP: EA) battery. Pain Med 2010;11:1546-1553.

84 Ahola K, Honkonen T, Isometsä E, Kalimo R, Nykyri E, Aromaa A, Lönnqvist J: The relationship between job-related burnout and depressive disorders - results from the Finnish Health 2000 Study. J Affect Disord 2005;88: 55-62.

85 Murck H: Atypical depression spectrum disorder - neurobiology and treatment. Acta Neuropsychiatr 2003;15:227-241.

86 Kudielka BM, Wüst S: Human models in acute and chronic stress: assessing determinants of individual hypothalamus-pituitaryadrenal axis activity and reactivity. Stress 2010;13:1-14.

87 MacCallum RC, Zhang S, Preacher KJ, Rucker DD: On the practice of dichotomization of quantitative variables. Psychol Methods 2002; 7:19-40.

88 Boomsma A: Robustness of LISREL against small sample sizes in factor analysis models; in Jöreskog GK, Wold H (eds): Systems under Indirect Observations, Causality, Structure, Prediction: Part 1. Amsterdam, North Holland, 1982, pp 149-173.

89 Bentler PM, Chou CP: Practical issues in structural modeling. Sociol Methods Res 1987;16:78-117.

90 Westland JC: Lower bounds on sample size in structural equation modeling. Electron Commer Res Appl 2010;9:476-487.

91 Iacobucci D: Structural equation modeling: fit indices, sample size, and advanced topics. J Consum Psychol 2010;20:90-98.

$\$ 92$ Little TD, Cunningham WA, Shahar G, Widaman KF: To parcel or not to parcel: exploring the question, weighing the merits. Struct Equ Modeling 2002;9:151-173.

93 MacCallum RC, Austin JT: Applications of structural equation modeling in psychological research. Ann Rev Psychol 2000;51:201-226.

$\$ 94$ Sarrazin P, Vallerand R, Guillet E, Pelletier L, Cury F: Motivation and dropout in female handballers: a 21-month prospective study. Eur J Sport Psychol 2002;32:395-418.

95 Comrey AL, Lee HB: A First Course in Factor Analysis. Hillsdale, Erlbaum, 1992.
Hair Cortisol, Perceived Stress and Depressive Symptoms
Neuropsychobiology 2013;68:100-109 DOI: $10.1159 / 000351735$ 